\title{
El Programa de Interrupción Legal del Embarazo en la Ciudad de México: experiencias del personal de salud
}

\author{
Claudia Díaz-Olavarrieta, ${ }^{1}$ Vanessa M. Cravioto, ${ }^{1}$ Aremis Villalobos, ${ }^{1}$ \\ Natalia Deeb-Sossa, ${ }^{2}$ Laura García $^{3}$ y Sandra G. García ${ }^{4}$
}

Forma de citar Díaz-Olavarrieta C, Cravioto VM, Villalobos A, Deeb-Sossa N, García L, García SG. El Programa de Interrupción Legal del Embarazo en la Ciudad de México: experiencias del personal de salud. Rev Panam Salud Publica. 2012;32(6):399-404.

RESUMEN Objetivo. Identificar las percepciones y opiniones del personal proveedor de servicios de aborto en la Ciudad de México, a tres años de la implementación de la reforma de la ley sobre aborto electivo.

Métodos. Se realizaron 19 entrevistas en profundidad entre febrero y junio de 2010 al personal de salud adscrito al Programa de Interrupción Legal del Embarazo (ILE) en una clinica y un hospital del Distrito Federal. Se recabó información sobre datos sociodemográficos, formación profesional y experiencia con provisión de servicios.

Resultados. Algunos participantes consideraron un acierto la gratuidad del servicio porque permite el acceso de mujeres de escasos recursos, en tanto que otros percibieron negativamente la exención del pago, a diferencia de otros procedimientos ginecobstétricos del sector salud. La objeción de conciencia prevaleció entre el personal de salud de ingreso reciente, lo cual podría atribuirse a su desconocimiento sobre lineamientos legales y técnicos del programa de ILE. Existe ambivalencia entre el personal por la no aceptación de un método anticonceptivo post-aborto por parte de algunas mujeres, lo que se percibe como un factor de reincidencia importante en los servicios de aborto legal.

Conclusiones. El personal de salud ostenta opiniones divididas y ambivalentes respecto al derecho de las mujeres a interrumpir un embarazo. Analizar experiencias y opiniones del personal de salud sobre la ley de ILE permitirá tener una línea basal sobre el tema y estudios futuros lograrán documentar cambios y retrocesos en la aprobación de dicha ley en México.

Palabras clave Aborto legal; personal de salud; derechos de la mujer; salud reproductiva; salud de la mujer; México.

América Latina y el Caribe cuentan con un marco jurídico restrictivo sobre el aborto. Chile y Nicaragua lo prohíben sin excepción, aunque este último lo

\footnotetext{
1 Centro de Investigación en Salud Poblacional, Instituto Nacional de Salud Pública, México. La correspondencia se debe dirigir a Claudia Díaz-Olavarrieta, Correo electrónico: colavarrieta@correo.insp.mx

2 Universidad de California, Davis, Estados Unidos.

3 Secretaría de Salud, Gobierno del Distrito Federal, México.

4 The Population Council, México.
}

permitía por razones terapéuticas hasta 2007. En Argentina y Ecuador es legal cuando la vida de la mujer está en peligro, o cuando el embarazo es producto de una violación a una mujer que presenta retraso mental o demencia. Brasil y Panamá lo contemplan en caso de malformaciones congénitas. Belice y Bolivia permiten interrumpir un embarazo para proteger la salud de la mujer. En la mayor parte de la Región, el aborto no es punible cuando la vida de la mujer corre peligro —en 10 países, Guatemala y Haití como ejemplos, esta es la única causal aprobada. En Uruguay y San Vicente y las Granadinas existe la causal de motivos socioeconómicos. El aborto sin restricciones solo está legalizado en ocho países, Cuba y Puerto Rico entre ellos (1).

En la capital de México, la despenalización del aborto durante las primeras 12 semanas de gestación (abril de 2007) 
fue resultado de dos reformas legislativas previas. La primera ocurrió en el año 2000, al firmarse una iniciativa para legalizar el aborto en casos de malformación congénita o cuando el embarazo constituyera un riesgo para la vida de la mujer (2), y la segunda en 2003, cuando la Asamblea Legislativa del Distrito Federal aprobó reformas al Código Penal - permitiendo a las mujeres acceder a servicios de aborto legal bajo una gama más amplia de causales de ley- y modificó la Ley de Salud para el Distrito Federal. Con base en estos cambios, la Secretaría de Salud del gobierno del Distrito Federal (SSDF) aprobó y publicó los lineamientos para regular la prestación de servicios de aborto legal en instituciones públicas y privadas del Distrito Federal (DF) (3).

Los lineamientos de prestación de servicios estipulan que la SSDF ofrecerá la interrupción legal del embarazo (ILE) gratuitamente a mujeres residentes del DF sin seguridad social. Aquellas radicadas en otros estados, o en la ciudad capital, y afiliadas a algún esquema público de salud, accederán a la atención pagando una cuota de recuperación (3-5), excepto en tres clínicas designadas para provisión gratuita de servicios de aborto. Las menores de edad deberán ir acompañadas por alguno de los padres o tutor legal, y todas deberán firmar un formulario de consentimiento informado para recibir la atención. Asimismo, la SSDF deberá disponer permanentemente de personal no objetor de conciencia (6) para proporcionar servicios, respetando la postura de los médicos objetores.

Después de la reforma, la SSDF implementó el primer programa público de prestación de servicios de aborto legal en México. A la fecha se han realizado 78544 procedimientos (7). Como consecuencia de la reforma en el DF, 17 estados modificaron sus constituciones locales para proteger la vida desde la concepción (8).

Desde la despenalización, el proceso administrativo para solicitar servicios de ILE se simplificó. Generalmente el primer contacto se realiza en el área de recepción, donde se explica el servicio y se registran datos personales, incluida la fecha de la última menstruación para determinar la edad gestacional y programar una cita subsecuente. Durante la primera consulta, una enfermera o trabajadora social otorga una sesión de consejería sobre los dos métodos dis- ponibles (médico o quirúrgico) y las opciones anticonceptivas posaborto. Las sesiones de consejería son individuales, aunque algunas clínicas ofrecen sesiones grupales debido al enorme número de mujeres que solicitan servicios (9).

La edad gestacional se confirma mediante ultrasonido realizado en la primera cita. Tras la fase de consejería, y dependiendo de su edad gestacional, la mujer elige el procedimiento. La SSDF ofrece el método quirúrgico a mujeres con edad gestacional de entre 9 y 12 semanas, y aborto con medicamentos a mujeres con hasta 64 días de gestación (10). El personal de trabajo social realiza el estudio socioeconómico para determinar el costo del procedimiento, basándose en el "tabulador de cobro de derechos por los servicios médicos" de la SSDF (3). Los lineamientos establecen que la consejería y la provisión de métodos anticonceptivos, excepto el dispositivo intrauterino (DIU) y la ligadura de trompas (11), estén a cargo primordialmente del personal de enfermería (3). Las trabajadoras sociales proporcionan consejería para reducir la carga de trabajo de las enfermeras.

Los resultados de un análisis de las experiencias y actitudes de quienes proveen servicios de aborto legal podrían contribuir a mejorar la calidad de la atención, y aportar información valiosa para desarrollar políticas públicas sobre salud reproductiva en países como México que, salvo la ciudad capital, cuentan con legislación restrictiva sobre el aborto (11-14). Siete meses después de la despenalización del aborto en el DF, se realizó un estudio cualitativo sobre conocimientos y actitudes de ginecobstetras, enfermeras, trabajadoras sociales y tomadores de decisión en la SSDF, quienes aportaron perspectivas diversas sobre la nueva ley (9).

A partir del análisis de opiniones y experiencias de proveedores de servicios de aborto adscritos al Programa de ILE, el presente trabajo se propone identificar las percepciones del personal que participa en la prestación de estos servicios en la Ciudad de México, a tres años de la implementación de la reforma de la ley sobre aborto electivo. Los resultados fueron obtenidos de entrevistas cara a cara con personal de salud, como parte del componente cualitativo de un proyecto más extenso —que combinó técnicas cuantitativas y cualitativasdel Departamento de Salud Reproduc- tiva de la Organización Mundial de la Salud (OMS).

\section{MATERIALES Y MÉTODOS}

Entre febrero y junio de 2010 se realizó un estudio cualitativo en una clínica y un hospital de la Ciudad de México. Ambos establecimientos concentran el $56 \%$ de todos los procedimientos de ILE realizados en los servicios públicos de salud (15).

Se entrevistó a una muestra por conveniencia de 10 proveedores de salud en la clínica y 9 en el hospital. Se diseñó una guía semiestructurada integrando datos demográficos, formación académica y siete ejes temáticos para explorar opiniones y experiencias del personal sobre solicitud y acceso de las mujeres a la ILE: i) opinión de la nueva ley sobre aborto, ii) descripción de servicios de aborto legal de la SSDF, iii) aceptabilidad de métodos de aborto, iv) capacitación en procedimientos, v) percepción sobre limitaciones enfrentadas dentro del Programa de ILE, vi) fortalezas y debilidades de los servicios en la SSDF y vii) recomendaciones para mejorar el Programa de ILE.

Dos entrevistadoras con experiencia en metodología cualitativa fueron capacitadas para realizar las entrevistas. Se asignó un espacio privado para garantizar la confidencialidad y se obtuvo de los entrevistados consentimiento informado por escrito e individual. Las entrevistas fueron grabadas y transcritas textualmente. Para el análisis se empleó la teoría interpretativa, mediante la técnica de análisis temático, y a través del programa Atlas.ti (5.0.67) se realizó una codificación abierta, con base en categorías elaboradas de forma mixta: categorías de análisis inicial, a partir de la guía de entrevista, y categorías emergentes detectadas en las entrevistas (16-18). El estudio fue aprobado por las Comisiones de Ética e Investigación de la OMS y del Instituto Nacional de Salud Pública de México (INSP), y autorizado en los dos establecimientos de salud participantes en la investigación.

\section{RESULTADOS}

\section{Características demográficas}

Dieciséis de los 19 participantes eran mujeres de entre 20 y 51 años de edad; ocho tenían estudios universitarios, dos 
en medicina general, tres en enfermería y obstetricia y tres en trabajo social; cuatro con especialidad médica (tres en ginecobstetricia y uno en anestesiología) y siete con entrenamiento técnico (cuatro en enfermería y tres en trabajo social); 14 reportaron más de un año trabajando en el programa de ILE y cinco eran de reciente incorporación ( $<1$ año) (cuadro 1$)$.

Del análisis realizado surgieron los siguientes seis temas: opinión sobre legalización del aborto hasta las 12 semanas de gestación en el Distrito Federal, despenalización del aborto en otros estados de México, cuota única de recuperación en servicios de ILE, provisión de servicios de ILE en centros de salud, objeción de conciencia y percepciones sobre aborto legal asociadas con la capacitación recibida.

\section{Opinión sobre la ley de ILE}

La mayoría de los participantes consideró que los servicios de aborto legal inciden en la disminución de la mortalidad materna por abortos sépticos o mal practicados, y facilitan el acceso a los servicios, principalmente a mujeres pobres. Cinco informaron tener una pos-

CUADRO 1. Características sociodemográficas y años de ejercicio profesional de personal de la Secretaría de Salud del Distrito Federal (SSDF) que provee servicios de aborto en la Ciudad de México, México, 2010

\begin{tabular}{|c|c|c|}
\hline Característica & No. & $\%$ \\
\hline \multicolumn{3}{|l|}{ Grupo etario (años) } \\
\hline $20-30$ & 4 & 21,1 \\
\hline $31-40$ & 10 & 52,6 \\
\hline $41-50$ & 4 & 21,1 \\
\hline$\geq 51$ & 1 & 5,3 \\
\hline \multicolumn{3}{|l|}{ Sexo } \\
\hline Hombre & 3 & 15,8 \\
\hline Mujer & 16 & 84,2 \\
\hline \multicolumn{3}{|l|}{ Escolaridad } \\
\hline Técnica & 7 & 36,8 \\
\hline Licenciatura & 8 & 42,1 \\
\hline Especialidad & 4 & 21,1 \\
\hline \multicolumn{3}{|l|}{ Profesión } \\
\hline Enfermero & 6 & 31,6 \\
\hline Trabajador social & 7 & 36,8 \\
\hline Médico ginecobstetra & 3 & 15,8 \\
\hline Médico anestesiólogo & 1 & 5,3 \\
\hline Médico general & 2 & 10,5 \\
\hline \multicolumn{3}{|c|}{ Años de antigüedad en SSDF } \\
\hline $1-10$ & 12 & 63,2 \\
\hline$\geq 11$ & 7 & 36,8 \\
\hline \multicolumn{3}{|l|}{ Años de antigüedad en } \\
\hline \multicolumn{3}{|l|}{ Programa de ILE ${ }^{a}$} \\
\hline$<1$ & 5 & 26,3 \\
\hline $1-3$ & 14 & 73,7 \\
\hline
\end{tabular}

Fuente: elaboración de las autoras.

a Interrupción legal del embarazo.

tura ambivalente respecto a la legislación; [aun cuando beneficia a las mujeres que desean interrumpir un embarazo] la mayoría de los entrevistados favoreció el aborto por causas "extremas", como violación, malformaciones congénitas y riesgos a la salud y la vida de la mujer.

\begin{abstract}
"Vienen porque no les funcionó el método, otras porque ya no tienen dinero; muchas pacientes es por violación también, cada una tiene su razón por la cual viene y lo respeto." 30 años de edad, enfermera (BV-P03).
\end{abstract}

Algunos participantes expresaron que la ILE puede tornarse en un método anticonceptivo, especialmente entre mujeres que no adoptan un método post-aborto, circunstancia que consideran facilita abortos repetidos.

\begin{abstract}
"Por irresponsabilidad, porque es algo que no les está costando. Algunas sí son muy cortantes y dicen 'no quiero un método', otras dicen 'es que ya no tengo pareja', pero a pesar de que les decimos que se lo lleve, que lo use y no vuelva a pasar por lo mismo, a veces dicen 'no, no' y se van así, sin método." 37 años de edad, ginecobstetra (BV-P09).
\end{abstract}

\section{Carácter privativo de la ILE}

Los participantes asociaron la exclusividad de la ley del DF con sobresaturación de servicios, debido a la gran demanda de atención y la insuficiencia de recursos materiales y humanos para satisfacerla.

\footnotetext{
"Sí, es muy desgastante que tengas que estar batallando, que no tengas material, es muy estresante porque estás a la espera de ver a qué horas me lo dan y ya tengo trabajo aquí." 34 años de edad, enfermera (BV-P01).
}

Los participantes favorecieron la legislación sobre aborto en el resto del país para replicar la reforma del DF, pues evitaría el desplazamiento de mujeres desde otros estados, el cual es oneroso para ellas e incrementa la carga de trabajo en los servicios. Al respecto, del total de procedimientos de ILE realizados hasta marzo de 2010, 25\% correspondía a mujeres residentes en otros estados (11).

“Qué malo que solamente en el Distrito Federal, porque estamos cargando con el gasto de todas las mujeres que solicitan la intervención, del punto de la Repú- blica que vengan, y eso genera gastos para el Estado." 51 años de edad, trabajadora social (BV-P05).

\section{Gratuidad del servicio de aborto legal}

El personal de la clínica manifestó que la gratuidad permite a mujeres de escasos recursos acceder a servicios, pero consideró que la exención de pago para quienes tienen algún esquema de seguridad social o residen en otras entidades, repercute negativamente en la calidad de la atención, generando sobrecarga de trabajo y carencia de recursos materiales y humanos.

Los participantes expresaron que debería establecerse una cuota única de recuperación o eximir del pago según el decil de ingreso, determinado por un estudio socioeconómico previo a la provisión de servicios, para paliar el desabastecimiento de recursos dentro del programa.

\footnotetext{
"Sí, habría que pagar algo, acuérdate que lo que nos cuesta lo cuidamos, y lo que no nos cuesta, 'no me costó, no tengo por qué cuidarlo', entonces hay pacientes que ya vienen de tercera vez, y si yo les cobrara pues lo pensarían mejor." 42 años de edad, anestesiólogo (BV-P02).
}

El personal del hospital aseguró que la gratuidad solo beneficia a mujeres residentes del DF sin seguridad social. Las mujeres radicadas en otros estados o en la ciudad capital, pero afiliadas a algún esquema público de salud, pagan una cuota basada en un estudio socioeconómico elaborado por personal de trabajo social.

\section{Transferencia de servicios de ILE al primer nivel de atención}

Para el personal de la clínica, referir servicios de ILE al primer nivel de atención, particularmente la aspiración manual endouterina (AMEU), no es ideal porque la infraestructura y los recursos humanos no son equiparables con aquellos disponibles en hospitales para resolver complicaciones quirúrgicas que pudieran derivarse del procedimiento. Consideró que ofrecer aborto médico sí es pertinente, porque el procedimiento se separa de las funciones del personal al ponerlo en manos de las mujeres, quienes reciben capacitación durante la consejería para autoadministrar el misoprostol-solo, vía bucal. 
"La responsabilidad prioritaria es en un hospital, puesto que tiene quirófanos y tienen todo para hacerlo de manera más viable, nuestra área es pequeña, ya tenemos una demanda excesiva, pero aun así seguimos trabajando." 34 años de edad, enfermera (BV-P07).

El personal del hospital consideró positiva la migración de servicios de aborto a clínicas, porque al ampliar la cobertura de atención especializada se reduce la sobrecarga de trabajo en hospitales, donde también se realizan otros procedimientos ginecobstétricos.

\section{Objeción de conciencia}

Según los lineamientos de ILE, la objeción de conciencia es un derecho exclusivo de los médicos, pero en ocasiones el personal paramédico y administrativo también se puede asumir como objetor. Los participantes manifestaron que esta ley permite decidir si se ha de realizar o no un procedimiento, basándose en principios personales; sin embargo, el código de procedimientos de la SSDF señala que todo el personal debe participar en los programas de la institución por pertenecer a los servicios públicos de salud o, en su caso, referir a las mujeres a otro sitio para que reciban la atención solicitada (3).

La mayoría de los participantes mencionó que la objeción de conciencia está determinada por cuestiones culturales y religiosas, así como por desinformación sobre salud reproductiva. Expresaron que algunos médicos sostienen un doble discurso, porque rehúsan la práctica del aborto en el ámbito público pero la efectúan en la esfera privada por ser lucrativa.

\begin{abstract}
“Culturalmente es un proceso difícil de vencer, porque la gente está acostumbrada a golpear con una mano y a pedir con la otra. Aquí satanizan el programa, pero cuando han llegado a necesitarlo acuden a él, entonces los objetores dicen 'sí, mira, estoy en contra del programa pero, oye, échale la mano a mi sobrina ¿no?'" 37 años de edad, ginecobstetra (BV-P09).
\end{abstract}

\section{Capacitación previa sobre aborto legal}

La mitad de los participantes no tenía experiencia en procedimientos de aborto legal hasta su incorporación al Programa de ILE. El personal médico mencionó

que su entrenamiento cubrió aspectos técnicos sobre manejo del legrado uterino instrumental, pero no recibió suficiente capacitación teórica y práctica sobre procedimientos de AMEU/aspiración eléctrica y aborto médico.

El personal de salud que participó desde el inicio en el Programa de ILE fue capacitado por la SSDF y organizaciones de la sociedad civil sobre marco legal y esquemas terapéuticos; en contraste, los informantes con menor tiempo en el programa señalaron no haber accedido a actividades de capacitación, sino que aprendieron los procesos mediante información proporcionada por personal a cargo de la atención y por la práctica al rotar en distintas áreas del servicio.

\begin{abstract}
"Las capacitaciones ya fueron viniendo conforme se fue estableciendo lo del programa, la licenciada que aquí nos está capacitando a nosotras, enfermeras, ya nos iba diciendo cómo preparar los equipos, cómo íbamos a atender a las pacientes, nos fue diciendo, un día inesperado llegaron pacientes y a quirófano, entonces ya ahí conforme fueron pasando las pacientes ya fue como se fue implementando todo de filtro, admisión, recuperación, quirófano." 30 años de edad, enfermera (BV-P03).
\end{abstract}

Se observaron discrepancias entre las percepciones de los proveedores de reciente incorporación y los que fueron capacitados para participar en el programa. Los primeros mostraron mayor ambivalencia y oposición a brindar servicios de aborto legal, mientras que los segundos poseen mayor conocimiento del procedimiento y expresan más empatía hacia las mujeres.

\section{DISCUSIÓN}

Estudios de opinión entre ginecobstetras en México y otros países de América Latina sostienen que estos profesionales tienden a favorecer el aborto según las circunstancias causales, frecuentemente muestran desconocimiento acerca del marco legal para proveer estos servicios y son ambivalentes respecto al derecho de las mujeres a decidir sobre un embarazo no deseado. Este resultado es similar al reportado en encuestas de opinión pública sobre aborto en población abierta en México $(19,20)$. Por otro lado, se ha documentado que el personal de salud informado sobre la legislación favorece más el aborto electivo y tiene mayor disposición a proporcionar tales servicios (21).

La reforma legislativa en Ciudad de México, considerada una "isla de libertades" (22), constituye un estudio de caso. El personal responsable de la provisión de servicios trabajó bajo presión para adoptar los protocolos terapéuticos basados en evidencia. El repentino cambio en la legislación repercutió en las prácticas del personal de salud, quien enfrentó un dilema al reflexionar su postura ideológica frente al aborto electivo.

El Programa de ILE se percibe como un servicio que permite a las mujeres, especialmente las de escasos recursos, acceder a servicios de aborto seguro, pero se piensa que los lineamientos deberían estipular el número de veces que puede solicitarse la atención. Algunos opinan que el programa es empleado como método anticonceptivo, aun cuando datos publicados sobre abortos reiterados en la SSDF informan una tasa de repetidoras inferior al 2\% (11).

Esta postura ambivalente asociada a la ILE se atenúa por la aceptación del aborto bajo determinadas causales: las participantes, principalmente enfermeras y trabajadoras sociales, consideran justificable solicitar servicios de aborto solo en casos extremos. Un estudio realizado entre personal de salud siete meses después del inicio del Programa de ILE refirió la misma ambivalencia: si bien se reconoció que la ley beneficiaría a las mujeres, se consideraba que algunas no tomaban seriamente la necesidad de adoptar anticonceptivos post-aborto y se mostró cierto malestar en relación a las mujeres que recurrían a abortos repetidos (9).

La gratuidad del servicio se percibe como un acierto porque reduce las barreras de acceso a servicios de aborto seguro, pero en la clínica se considera un factor que disminuye la calidad de la atención relacionado con la sobrecarga de trabajo. Asimismo, se piensa que la gratuidad incentiva la no adopción de un método anticonceptivo post-aborto y también la reincidencia. Un estudio reportó que $38 \%$ de los proveedores se oponían a proporcionar servicios de aborto de manera gratuita, porque se deberían compensar los costos elevados del programa y crear un elemento de disuasión para evitar abusos (9). El mecanismo sugerido por los participantes en el presente estudio fue implementar un sistema de cuotas en el resto de los 
servicios públicos de salud que proporcionan la atención. En contraste, otro estudio cualitativo que documentó las experiencias de 10 mujeres que tuvieron abortos múltiples, encontró que estas necesitaban más apoyo en el manejo de su fertilidad, y por tanto la consejería debería enfocarse en las necesidades de cada paciente, y no en la prevención de abortos repetidos que las estigmatiza entre los proveedores (23).

El personal de salud aprueba la objeción de conciencia, aunque la vincula con un doble discurso empleado por algunos médicos que la invocan para excusarse de la práctica del aborto en el sector público. Un estudio (9) resaltó que los objetores no necesariamente tienen objeciones morales para su práctica, sino que intentan evitar la sobrecarga de trabajo. En el presente estudio los participantes refirieron, anecdóticamente, que algunos médicos definidos como "objetores" realizan abortos en el ámbito privado. Comparativamente, otro estudio sobre personal médico y aborto por violación en México reportó que los médicos en el sector público pueden generar barreras para ofrecer el servicio, pero estas mismas barreras desaparecen o son superadas en el ejercicio de la medicina privada (24).

El personal de salud participante en la fase inicial del Programa de ILE fue capacitado por la SSDF, así como por organizaciones de la sociedad civil dedicadas a la promoción y defensa de derechos sexuales y reproductivos. La disparidad de formación entre proveedores que han sido instruidos para colaborar en el programa y aquellos de reciente incorporación, incide en sus percepciones. Los primeros tienen mayor conocimiento sobre los procedimientos y expresan mayor empatía hacia las mujeres, mientras que los segundos muestran mayor ambivalencia y resistencia a brindar servicios de aborto legal, en opinión de algunos, por no estar debidamente sensibilizados sobre los derechos reproductivos de las mujeres. La formación médica tradicional ofrece pocos elementos para comprender la diversidad de motivos por los cuales las mujeres interrumpen un embarazo, y ubicar al aborto como un problema de salud pública, inequidad social, acceso a servicios y derechos reproductivos $(12,13)$.

Entre los participantes recién incorporados al programa, es más pronunciado el malestar sobre mujeres que no adoptan un método anticonceptivo postaborto o repiten el procedimiento. Sin embargo, los resultados de un estudio cualitativo entre mujeres que accedieron a servicios de ILE se contraponen a esta percepción. La satisfacción de las usuarias de servicios públicos de salud podría aumentar si se ampliara la consejería sobre anticoncepción post-aborto, elemento central del Programa de ILE $(9,12,25)$.

El personal de salud percibe positivamente el programa porque disminuye la mortalidad materna por abortos sépticos o mal practicados. Asimismo, la ley ha promovido el respeto y la concientización de las necesidades y demandas de las mujeres, incluso cuando todavía persiste la postura de favorecer más el aborto legal cuando existe una causal extrema. El tema de las "repetidoras" continúa inquietando a los proveedores, quienes opinaron que la ley debería establecer un límite de acceso a la atención, así como la obligatoriedad de emplear un método anticonceptivo post-aborto. La principal limitante metodológica del presente estudio es que la muestra de 19 proveedores de salud es reducida, y no permite hacer generalizaciones a todo el personal de salud asociado con la prestación de servicios de aborto en el DF.

El personal de salud de los servicios de ILE ostenta opiniones divididas y ambivalentes respecto al derecho de decidir sobre un embarazo no deseado. A futuro, será necesario reforzar la legalidad del procedimiento y el derecho de las mujeres para acceder a servicios de interrupción del embarazo, así como fortalecer los programas de planificación familiar para reducir la tasa de embarazos no planeados. Analizar experiencias y opiniones sobre el aborto legal permitirá tener una línea basal sobre el tema, mientras que mediante estudios futuros se logrará obtener un registro amplio sobre cambios y retrocesos en la aprobación de la ley en México. El programa de aborto legal del DF tiene el potencial de convertirse en un modelo para servicios privados en el país y para futuros programas de aborto legal en otros estados de México y el resto de América Latina y el Caribe.

Agradecimientos. Las autoras agradecen a Olimpia Gómez por su colaboración en la recolección de datos y a todo el personal de salud del Gobierno del Distrito Federal que participó en las entrevistas.

Financiamiento. El presente trabajo fue financiado por el Programa Especial PNUD/UNFPA/OMS/Banco Mundial de Investigaciones, Desarrollo y Formación de Investigadores sobre Reproducción Humana.

\section{REFERENCIAS}

1. Lerner S, Guillaume A. Las adversas consecuencias de la legislación restrictiva sobre el aborto: argumentos y evidencias empíricas en la literatura latinoamericana. Trabajo presentado en el III Congreso de la Asociación Latinoamericana de Población (Córdoba, Argentina, 24-26 sep de 2008). Disponible en: http://www.alapop.org/2009/ima ges/DOCSFINAIS_PDF/ALAP_2008_ FINAL_197.pdf Acceso el 10 de noviembre de 2012.

2. Kulczycki A. Abortion in Latin America: Changes in Practice, Growing Conflict, and
Recent Policy Developments. Stud Fam Plann. 2011;42(3):199-220.

3. México, Secretaría de Salud del Gobierno del Distrito Federal (SSDF). Manual de procedimientos para la interrupción legal del embarazo en las unidades médicas. Ciudad de México: SSDF; 2008.

4. Madrazo A. The evolution of Mexico City's abortion laws: From public morality to women's autonomy. Int J Gynecol Obstet. 2009;106:266-9.

5. Sánchez-Fuentes ML, Paine J, Elliott-Buettner B. The decriminalization of abortion in
Mexico City: How did abortion rights become a political priority? Gend Dev. 2008;16:345-60. 6. México, Ley de Salud para el Distrito Federal. Disponible en: http://www.sma.df.gob. $\mathrm{mx} / \mathrm{sma} /$ links/download/biblioteca/ leyes_equidad/local/11_ley_salud_ df.pdf Acceso el 13 de noviembre de 2012.

7. Grupo de Información en Reproducción Elegida (GIRE). Cifras ILE, abril 2012. Disponible en: http://www.gire.org.mx/ index.php?option $=$ com_content\&vie $\mathrm{w}=$ article $\& \mathrm{id}=504 \% 3$ Acifras-ile-abril2012 \& catid $=166 \% 3$ Ainformacon- 
relevante\&Itemid=1397\&lang=es Acceso el 13 de noviembre de 2012.

8. Grupo de Información en Reproducción Elegida (GIRE). Reformas aprobadas a las constituciones estatales que protegen la vida desde la concepción/fecundación 2008-2011. Disponible en: http://www.gire.org.mx/ publica2/ReformasAbortoConstitucion Marzo14_2011.pdf Acceso el 13 de noviembre de 2012 .

9. Contreras X, Van Dijk M, Sánchez T, Sanhueza P. Experiences and Opinions of Health-Care Professionals Regarding Legal Abortion in Mexico City: A Qualitative Study. Stud Fam Plann. 2011;42(3):183-90.

10. Winikoff B, Dzuba IG, Creinin MD, Crowden WA, Goldberg AB, Gonzalez J, et al. Two distinct oral routes of misoprostol in mifepristone medical abortion: a randomized controlled trial. Obstet Gynecol. 2008;112(6): 1303-10.

11. Mondragón y Kalb M, Ahued Ortega A, Morales Velázquez J, Díaz-Olavarrieta C, Valencia Rodríguez J, Becker D, et al. Patient Characteristics and Service Trends Following Abortion Legalization in Mexico City, 200710. Stud Fam Plann. 2011;42(3):159-66.

12. Becker D, Díaz-Olavarrieta C, Juárez C, Garcia S, Sanhueza P, Harper C. Clients' perceptions of the quality of care in Mexico City's publicsector legal abortion program. Int Perspect Sex Reprod Health. 2011;37(4):191-201.

13. González de León-Aguirre D, Billings D, Ramírez-Sánchez R. El aborto y la educa- ción médica en México. Salud Publica Mex. 2008;(50)3:258-67.

14. Gasman N, Blandon M, Crane B. Abortion, social inequity, and women's health: Obstetrician-gynecologists as agents of change. Int J Gynecol Obstet. 2006;94(3):310-6.

15. México, Secretaría de Salud del Gobierno del Distrito Federal. Agenda Estadística, 2010. Disponible en: http://www.salud.df.gob. $\mathrm{mx} / \mathrm{ssdf} /$ info_salud/Pagina\%20Web\% 20 Agenda \% 20Estad \% C $3 \%$ ADstica $\% 202010 /$ inicio.html Acceso el 13 de noviembre de 2012.

16. Denzin N. Un punto de vista interpretativo. En: Denman C, Haro J, eds. Por los rincones. Antología de métodos cualitativos en la investigación social. Hermosillo, Sonora: El Colegio de Sonora; 2000.

17. Braun V, Clarke V. Using thematic analysis in psychology. Qual Res Psychol. 2006;3(2): 77-101.

18. Muñoz J. Análisis cualitativo de datos textuales con ATLAS.ti 5. Barcelona: Universitat Autónoma de Barcelona, 2005. Disponible en: http://uab.academia.edu/ JuanMu \% C3\% B1ozJusticia / Papers / 94666/Analisis_de_datos_textuales_con Atlas.ti_5 Acceso el 13 de noviembre de 2012.

19. Wilson K, García S, Díaz-Olavarrieta C, Villalobos-Hernández A, Valencia J, Sanhueza $\mathrm{P}$, et al. Public Opinion on Abortion in Mexico City after the Landmark Reform. Stud Fam Plann. 2011;42(3):175-82.
20. Valencia J, Wilson K, Diaz-Olavarrieta C, García S, Sánchez M. Public Opinion on Abortion in Eight Mexican States amid Opposition to Legalization. Stud Fam Plann. 2011;42(3): 191-8.

21. García S, Lara D, Goldman L. Conocimientos, actitudes y prácticas de los médicos mexicanos sobre el aborto: Resultados de una encuesta nacional. Gac Med Mex. 2003;139(S1):S91-102.

22. Diaz-Olavarrieta C. El aborto inducido en México. La ciudad capital como una "isla de libertades". En: Dides C, Benavente C, Sáez I, eds. Dinámicas políticas sobre aborto en Latinoamérica: Estudio de casos. Chile: FLACSO; 2011.

23. Weitz T, Kimport K. A need to expand our thinking about "repeat" abortions. Contraception. 2012;85(4):408-12.

24. Silva M, Billings D, García S, Lara D. Physicians' agreement with and willingness to provide abortion services in the case of pregnancy from rape in Mexico. Contraception. 2009;79(1):56-64

25. Van Dijk M, Arellano L, Arangure A, Toriz A, Krumholz A, Yam E. Women's experiences with legal abortion in Mexico City: A qualitative study. Stud Fam Plann. 2011;42(3):167-74.

Manuscrito recibido el 12 de enero de 2012. Aceptado para publicación, tras revisión, el 9 de septiembre de 2012.

ABSTRACT Objective. Identify the perceptions and opinions of people who provide abortion services in Mexico City, three years after implementation of elective abortion legal reforms.

\section{Mexico City's Legal Abortion Program: health workers' experiences}

Key words
Methods. Nineteen in-depth interviews of health workers assigned to the legal abortion programs at a clinic and a hospital in Mexico's Federal District were carried out between February and June of 2010. Information on sociodemographic data, professional training, and experience in providing services was collected.

Results. Some interviewees thought the provision of free services was beneficial because it allowed lower-income women to access this type of care, whereas others interviewed disapproved of the lack of fees, since other gynecological and obstetric health services have to be paid for. Conscientious objection prevailed among newly hired health workers, which can be attributed to their lack of knowledge about the legal abortion program's legal and technical guidelines. Some workers were ambivalent because they did not accept a postabortion contraception method used by some women, perceiving it to be a factor in significant repeated demand for legal abortion services.

Conclusions. Health workers evince divided and ambivalent opinions with regard to abortion rights. Analyzing their experiences and opinions will facilitate the creation of a baseline on the subject, and future studies will be able to document changes in and any lessening of approval for this law in Mexico.

Abortion, legal; health personnel; women's rights; reproductive health; women's health; Mexico. 